\title{
Unusual cause of hypoglycemia in a type 2 diabetic patient: panhypopituitarism
}

\author{
F. BALEANU, L. ICONARU, C. BOURMORCK, R. KARMALI \\ CHU BRUGMANN, BRUSSELS, BELGIUM
}

\section{Introduction}

Pituitary adenoma is a well-known cause of panhypopitutarism. The degree of hypopituitarism is variable ranging from a single hormone deficiency to a general disorder. The onset in most cases is in several months or even years, so it is often misrecognized and not adequately treated. The presenting symptoms are also extremely variable. Recurrent hypoglycemia due to growth hormone, adrenal and other counter regulatory hormone deficiency is a less well-known complication of hypopituitarism.

\section{Case}

We report on a 50-year-old patient of Moroccan origin, with insulin treated type 2 diabetes known for several years.

He presented with a 6 months history of hypoglycemia, hypotension, associated with loss of libido and erectile dysfunction, anorexia, general deterioration and weakness.

He had lost $20 \mathrm{~kg}$ over a 6-month period.

The insulin doses were diminished from $70 \mathrm{U} /$ day to $42 \mathrm{U} /$ day and the antihypertensive treatment was stopped due to repeated episodes of hypotension.

\section{Diagnostic Tests and Clinical Course:}

Laboratory results showed reduced free thyroxin with normal thyroid- stimulating hormone and diminished morning serum cortisol levels. Besides, testosterone, luteinizing hormone and insulin- like growth factor-1 levels were also reduced.

The pituitary stimulation tests $(\mathrm{TRH}, \mathrm{LHRH}$, Insulin induced hypoglycemia) revealed a delayed response in $\mathrm{TSH}$, a delayed and prolonged response in $\mathrm{LH}$, and no response in ACTH and $\mathrm{GH}$.
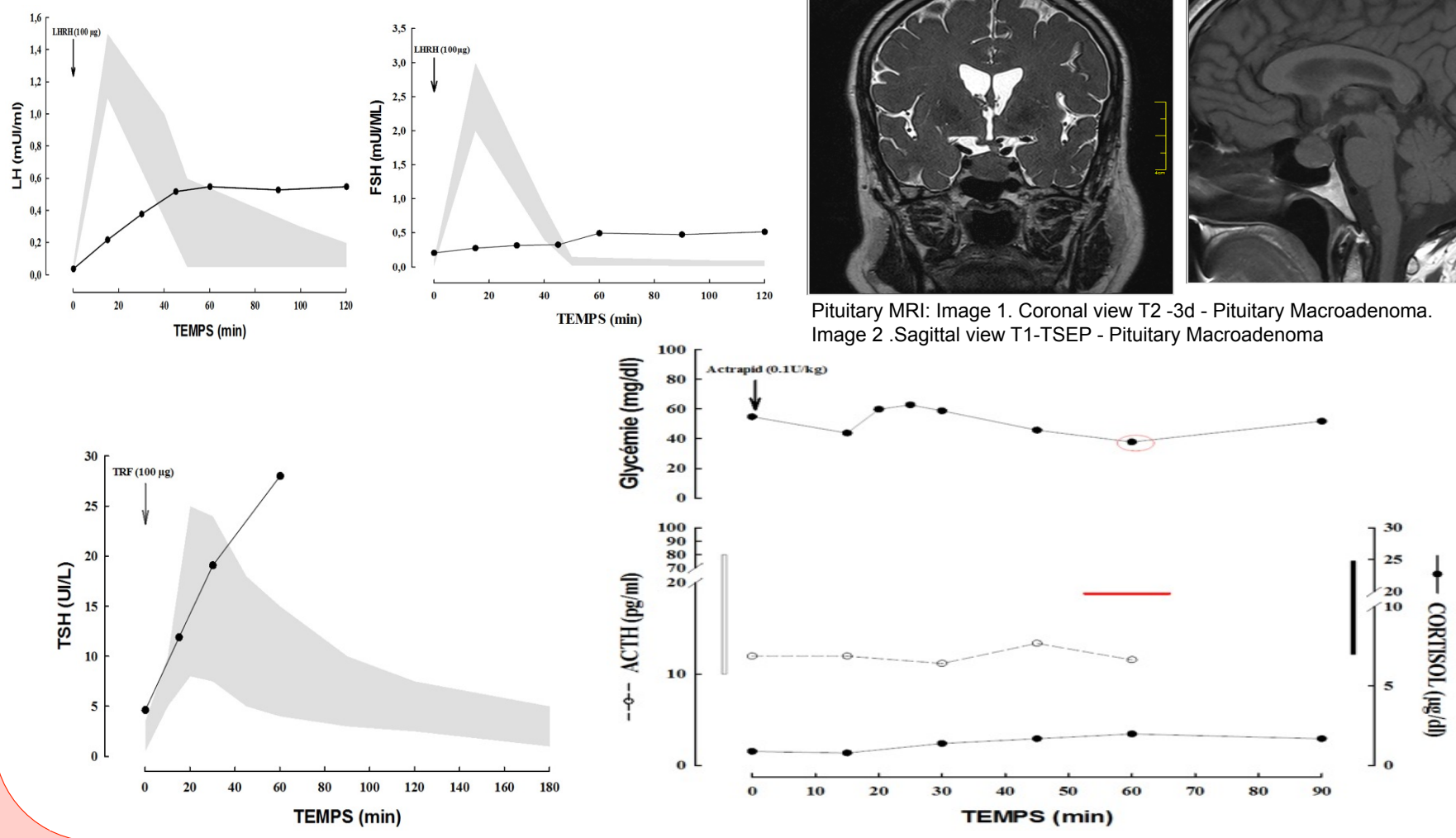

\section{Conclusion}

Hypoglycemia and erectile dysfunction in a diabetic patient should not overlook other causes than excessive treatment and diabetes.
A MRI of the sella turcica revealed a macro adenoma with a left deviation of the pituitary stalk and a minimal compression of optical chiasma.
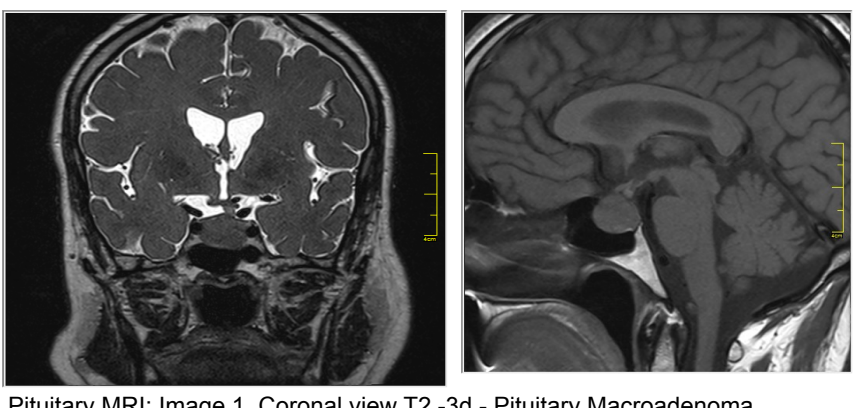

Pituitary MRI: Image 1. Coronal view T2 -3d - Pituitary Macroadenoma. Image 2 .Sagittal view T1-TSEP - Pituitary Macroadenoma 